\title{
SLOW FORCING IN THE PROJECTIVE DYNAMICS METHOD
}

\author{
M. A. Novotny ${ }^{a}$ M. Kolesik ${ }^{b}$ and P. A. Rikvold ${ }^{a, c}$ \\ a Supercomputer Computations Research Institute (SCRI), Florida State \\ University, Tallahassee, FL 32306-4130, U.S.A. \\ b Institute of Physics, Slovak Academy of Sciences, Dúbravská cesta 9, 84228 \\ Bratislava, Slovak Republic \\ ${ }^{\mathrm{c}}$ Center for Materials Research and Technology (MARTECH) and Department of \\ Physics, Florida State University, Tallahassee, FL 32306-4350, U.S.A.
}

\begin{abstract}
We provide a proof that when there is no forcing the recently introduced projective dynamics Monte Carlo algorithm gives the exact lifetime of the metastable state, within statistical uncertainties. We also show numerical evidence illustrating that for slow forcing the approach to the zero-forcing limit is rather rapid. The model studied numerically is the 3-dimensional 3-state Potts ferromagnet.
\end{abstract}

Key words: Monte Carlo, Projective Dynamics, Metastability

Preprint submitted to Elsevier Preprint

14 June 2021 
One of the most challenging hurdles to be overcome in computational materials science is finding a direct way to bridge the disparate time and length scales required in simulations of models of materials. The length scales go from the atomic to the size of the actual material sample. The shortest time scale in many instances can be associated with an inverse phonon frequency, and the longest time scale with the mean time before macroscopic material failure. One way of bridging these time and length scales is to devise better algorithms. Such algorithms need to increase computational efficiency by orders of magnitude in order to allow the desired material simulations to proceed on today's computers.

Although there are a number of efficient algorithms for the statics of model systems [1], the number of advanced algorithms for dynamics is more limited. These include the $n$-fold way algorithm [2], and its generalization, the Monte Carlo with Absorbing Markov Chains (MCAMC) algorithm [3].

Recently, the authors have devised an improved dynamical algorithm for models with discrete numbers of states at each lattice site. This algorithm is called the Projective Dynamics (PD) method [4]. The PD algorithm builds on previous work using projections onto slow variables in dynamical models $[5,6]$. The $\mathrm{PD}$ algorithm has been shown to allow simulations of the dynamics of systems such as the Ising model for very long times and large lattice sizes [7], requiring quite modest computer resources. It has also been shown to allow identification of physical quantities, such as activation volumes and Barkhausen volumes, in dynamical models of magnetic domain growth [8].

In this brief paper we concentrate on the use of the PD algorithm to obtain the average lifetime of the metastable state in a model system. In particular, we apply the PD algorithm to the escape from the metastable state of the three-dimensional 3-state Potts ferromagnet.

The 3-state Potts model in applied field $H$ has the Hamiltonian

$$
\mathcal{H}=-J \sum_{\langle i, j\rangle} \delta\left(\sigma_{i}, \sigma_{j}\right)+H \sum_{i}\left[\delta\left(0, \sigma_{i}\right)-\delta\left(1, \sigma_{i}\right)\right]
$$

where $\sigma_{i} \in\{0,1,2\}$ is the 'spin' at lattice site $i$. The first summation runs over all nearest-neighbor pairs on a simple-cubic lattice, and $J>0$ corresponds to ferromagnetic interactions. The second sum runs over all lattice sites, and it only changes the energies of spins in the states 0 and 1 . The system can be characterized by the concentrations $\left\{n_{0}, n_{1}, n_{2}\right\}, \sum n_{i}=1$, of spins in the three states.

The Monte Carlo simulation uses local updates at randomly chosen sites. We use simple cubic lattices of size $L^{3}=V$ with periodic boundary conditions. 
In this paper we use Glauber dynamics, and we measure the time in Monte Carlo Steps per Spin (MCSS). The simulation starts with all spins in state 0, with the temperature $T$ below the critical temperature $T_{\mathrm{c}}$. We measure the average lifetime $\tau$, defined as the average first-passage time to a configuration with half of the spins in the stable state, which here is state 1. Our Monte Carlo simulation can be viewed as a random walk which starts in the state with $n_{0}=V$ and goes until it reaches a state with $n=N=V / 2$, where we have dropped the subscript on $n=n_{1}$.

The spins in the current configuration can be divided into classes specified by the state $\sigma$ of the spin and by the numbers $\{a, b, 6-a-b\}$ of neighbors in the states $\{0,1,2\}$. Let $c_{a b}^{\sigma}\left(n_{0}, n_{1}, n_{2}\right)$ be the average concentration of spins in the class $\{\sigma, a, b\}$, conditional on the total concentrations $n_{i}$ of spins in state $i$. This average is obtained from repeated metastable simulations. The class concentrations are normalized such that $\sum_{\sigma a b} c_{a b}^{\sigma}\left(n_{0}, n_{1}, n_{2}\right)=1$ for all $\left\{n_{0}, n_{1}, n_{2}\right\}$. Let $p_{a b}^{\sigma \sigma^{\prime}}$ be the probability that a spin in the class $\{\sigma, a, b\}$ will flip to the state $\sigma^{\prime}$ when visited by the updating algorithm. We project the spin class concentrations onto bin $n$ by defining $c_{a b}^{\sigma}(n)=\sum_{n_{0}, n_{2}} c_{a b}^{\sigma}\left(n_{0}, n_{1}=n, n_{2}\right)$. The projected flip rates are defined in terms of the $c_{a b}^{\sigma}(n)$ and the flipping probabilities $p_{a b}^{\sigma \sigma^{\prime}}$ as:

$$
g(n)=\sum_{a b, \sigma \neq 1} c_{a b}^{\sigma}(n) p_{a b}^{\sigma 1}, \quad s(n)=\sum_{a b, \sigma \neq 1} c_{a b}^{1}(n) p_{a b}^{1 \sigma} .
$$

The rates $s(n)$ and $g(n)$ correspond to the shrinkage and growth rates of the stable phase, respectively. They depend on how the configurations are generated to obtain the $c_{a b}^{\sigma}(n)$. Here we obtain the configurations during simulation of escape from the metastable state, with and without the forcing described below.

Using standard methods from the theory of absorbing Markov chains [3,6], the mean lifetime $\tau$ and the total average time $h(n)$ (measured in MCSS, with $h(N)=0$ ) spent by the random walker in the state $n$ are given, as shown in the appendix, by

$$
\tau=\sum_{n=0}^{N-1} h(n), \quad h(n-1)=\frac{V^{-1}+s(n) h(n)}{g(n-1)} .
$$

An enhancement to the PD algorithm described above was presented in Ref. [4]. At any time, the system is only allowed to have the number of spins in the stable phase, $n$, strictly larger than a lower bound $n_{\min }(t)$. The simulation starts with $n_{\min }=-1$, and $n_{\min }$ is increased at a slow constant rate. The class concentrations $c_{a b}^{\sigma}(n)$ are measured during this forced escape and used to calculate $\tau$ from Eq. (3). In the appendix we prove that if the random walk does not 
hit the moving lower bound, the value of $\tau$ obtained with the PD algorithm is exact. Figure 1 shows the approach of $\tau$ to the slow-forcing limit as the forcing rates is lowered. Even at moderately high forcing rates the lifetime (with statistics between $10^{2}$ and $10^{3}$ escapes from the metastable state) is indistinguishable from the value at zero forcing rate. The forcing, however, decreases substantially the amount of computer time required to obtain $\tau$. In the conventional direct simulation approach, the necessary computer time increases exponentially with the depth of the metastable free-energy minimum. On the other hand, in the forced-escape method, the computer time is proportional to the depth of the free-energy minimum itself. In other words, the proposed method allows calculations of an exponentially hard problem in linear time. Although here we only present data for a temperature and field at which direct simulation is possible and the speed-up is only about $10^{2}-10^{3}$, the forced escape method allows one to obtain lifetimes many orders of magnitude longer than would be possible without forcing $[4,7]$.

In conclusion, we have obtained the average lifetime, $\tau$, of the metastable state of the 3-state three-dimensional Potts ferromagnet using forcing in the PD (Projective Dynamics) method. In the limit of zero forcing, we prove in the appendix that the value of $\tau$ obtained is exact. Substantial savings in computer time can be achieved using relatively moderate forcing rates without significantly affecting the obtained values of $\tau$.

\section{ACKNOWLEDGEMENTS}

This research was supported in part by U.S. NSF Grants 9520325 and 9871455 , and by the Florida State University through MARTECH and through SCRI (DOE Contract No. DE-FC05-85ER25000). MK was also supported by VEGA grant no. 2/4109/97. Supercomputer access provided by the DOE at NERSC.

\section{APPENDIX: Lifetimes from projective dynamics}

We prove that if there is no forcing, the lifetime obtained from the projective dynamics algorithm is exact. Of course, there will still be statistical uncertainties in the lifetime obtained from any finite length simulation. The proof is given for the model under consideration in this paper, the three-dimensional three-state Potts model. Furthermore, we choose state 1 as the slow variable on which we project the dynamics. The proof can easily be generalized to other models and dimensions as long as the number of states in the model is finite. The notation we use is given below.

- $V$ - total number of spins in the system.

- $S=\bigcup_{n=0}^{V} S_{n}$ - partition of the state space $S$ of the model into disjoint 
subspaces where $S_{n}$ contains all microstates with exactly $n$ spins in state 1 . - $[n, i]$ — label for the $i$ th state in $S_{n}, i=1,2, \ldots,\left|S_{n}\right|$, where $|\cdots|$ denotes the number of elements in the partition.

- $C_{a b}^{\sigma}(n, i)$ - the number of those spins of the microstate $[n, i]$ which are in state $\sigma$ and have $a$ and $b$ neighbors in states 0 and 1, respectively. Thus, $\sum_{a b \sigma} C_{a b}^{\sigma}(n, i)=V$ for each $[n, i]$.

- $c_{a b}^{\sigma}(n, i)=C_{a b}^{\sigma}(n, i) / V$ denotes concentrations.

- $p_{a b}^{\sigma \sigma^{\prime}}$ - probability that a chosen spin in state $\sigma$ having $a, b$ neighbors in states 0 and 1 will flip to state $\sigma^{\prime}$.

Consider direct Monte Carlo measurement of the mean lifetime $\tau$ without forcing. This can be accomplished by setting $n_{\min }=-1$ at all times. Start an escape from the state $[0,1]$ and continue the simulation until the Monte Carlo "random walker" reaches one of the absorbing states $\left[n_{\text {stop }}, j\right]$ with $j=1,2, \ldots,\left|S_{n_{\text {stop }}}\right|$. A total number $N_{\text {esc }}$ of independent escapes are realized. Denote by $W(n, i)$ the number of visits in the state $[n, i]$ generated by this repeated measurement (counting rejected Monte Carlo moves as new visits in the same state). Then

$$
h(n)=\frac{1}{V} \sum_{i=1}^{\left|S_{n}\right|} W(n, i) / N_{\mathrm{esc}}
$$

is the average (sojourn) time spent in $S_{n}$, in units of Monte Carlo Steps per Spin (MCSS). The mean lifetime is obtained by

$$
\tau=\sum_{n=0}^{n_{\text {stop }}-1} h(n)=\frac{1}{V} \sum_{n=0}^{n_{\text {stop }}-1} \sum_{i=1}^{\left|S_{n}\right|} W(n, i) / N_{\mathrm{esc}} .
$$

One can imagine that each random walker generates an oriented "world line" starting in $[0,1]$ and ending in some $\left[n_{\text {stop }}, j\right]$, because the transitions are possible only between neighboring $S_{n}$ and $S_{n \pm 1}$ subspaces. Therefore, an "equation of continuity" holds for each $n<n_{\text {stop }}$ :

$$
N_{n \rightarrow n+1}=N_{\mathrm{esc}}+N_{n+1 \rightarrow n}
$$

where $N_{\ell \rightarrow \ell^{\prime}}$ stands for the number of transitions between the subspaces with $\ell$ and $\ell^{\prime}$ spins in state 1 . Equation (6) can be written in terms of $W, C_{a b}^{\sigma}$ and $p_{a b}^{\sigma \sigma^{\prime}}$. To do this we observe that the probability that a particular attempted spin flip results in a move from $S_{n}$ to $S_{n+1}$ is $V^{-1} \sum_{a b} \sum_{\sigma \neq 1} C_{a b}^{\sigma}(n, i) p_{a b}^{\sigma 1}$, where the factor $V^{-1}$ represents the probability of choosing one particular spin. Consequently, 


$$
\begin{aligned}
& \frac{1}{V} \sum_{i=1}^{\left|S_{n}\right|} W(n, i) \sum_{a b} \sum_{\sigma \neq 1} C_{a b}^{\sigma}(n, i) p_{a b}^{\sigma 1}= \\
& N_{\mathrm{esc}}+\frac{1}{V} \sum_{i=1}^{\left|S_{n+1}\right|} W(n+1, i) \sum_{a b} \sum_{\sigma \neq 1} C_{a b}^{1}(n+1, i) p_{a b}^{1 \sigma}
\end{aligned}
$$

Since each $S_{n}$ must be visited at least $N_{\text {esc }}$ times, we can multiply the two terms involving sums by an identity and write the above equation as

$$
\begin{aligned}
& \frac{\sum_{j=1}^{\left|S_{n}\right|} W(n, j)}{\sum_{k=1}^{\left|S_{n}\right|} W(n, k)} \sum_{a b} \sum_{\sigma \neq 1} p_{a b}^{\sigma 1} \sum_{i=1}^{\left|S_{n}\right|} W(n, i) c_{a b}^{\sigma}(n, i)=N_{\mathrm{esc}} \\
& +\frac{\sum_{j=1}^{\left|S_{n+1}\right|} W(n+1, j)}{\sum_{k=1}^{\left|S_{n+1}\right|} W(n+1, k)} \sum_{a b} \sum_{\sigma \neq 1} p_{a b}^{1 \sigma} \sum_{i=1}^{\left|S_{n+1}\right|} W(n+1, i) c_{a b}^{1}(n+1, i) .
\end{aligned}
$$

In Eq. (8) one recognizes the average lumped class concentration

$$
c_{a b}^{\sigma}(n)=\sum_{i=1}^{\left|S_{n}\right|} W(n, i) c_{a b}^{\sigma}(n, i) / \sum_{k=1}^{\left|S_{n}\right|} W(n, k)
$$

which enters the definition of the global spin-flip rates, Eq. (2). We divide by $V N_{\mathrm{esc}}$ to express the sojourn times in units of MCSS. This gives

$$
h(n) \sum_{a b} \sum_{\sigma \neq 1} p_{a b}^{\sigma 1} c_{a b}^{\sigma}(n)=V^{-1}+h(n+1) \sum_{a b} \sum_{\sigma \neq 1} p_{a b}^{1 \sigma} c_{a b}^{1}(n+1),
$$

which leads directly to Eq. (3). Thus, in the absence of forcing, our formula for the lifetime becomes exact.

\section{References}

[1] R.H. Swendsen, J.S. Wang, and A.M. Ferrenberg, in The Monte Carlo Method in Condensed Matter Physics, edited by K. Binder and A. Baumgartner, second edition, p. 75 (Springer, Berlin, 1995).

[2] A.B. Bortz, M.H. Kalos, and J.L. Lebowitz, J. Comput. Phys. 17, 10 (1975).

[3] M.A. Novotny, Phys. Rev. Lett. 74, 1 (1995); 75, 1424(E) (1995); Comput. in Phys. 9, 46 (1995).

[4] M. Kolesik, M.A. Novotny, and P.A. Rikvold, Phys. Rev. Lett. 80, 3384 (1998).

[5] L.S. Schulman, J. Phys. A: Math. Gen. 13, 237 (1980). 
[6] J. Lee, M.A. Novotny, and P.A. Rikvold, Phys. Rev. E 52, 356 (1995).

[7] M. Kolesik, M.A. Novotny, P.A. Rikvold, and D.M. Townsley, in Computer Simulation Studies in Condensed-Matter Physics X, edited by D.P. Landau, K.K. Mon and H.-B. Schüttler (Springer, Berlin, 1998), p. 246.

[8] M. Kolesik, M.A. Novotny, and P.A. Rikvold, Mat. Res. Soc. Symp. Proc., preprint cond-mat/9801052, in press.

\section{Figure Captions}

Fig. 1. The measured average lifetime, $\tau$, is shown as a function of the inverse forcing rate. This figure illustrates that excellent values for $\tau$ are obtained even with moderately fast forcing. The figure is for $10^{2}-10^{3}$ escapes from the metastable state. To achieve uniform accuracy, more escapes are needed at high forcing rates. 